\title{
Effective dynamics and steady state of an Ising model submitted to tapping processes
}

\author{
A. Prados* and J. Javier Brey ${ }^{\dagger}$ \\ Física Teórica, Universidad de Sevilla, Apartado de Correos 1065, E-41080 Sevilla, Spain
}

(Received 3 July 2002; published 29 October 2002)

\begin{abstract}
A one-dimensional Ising model with nearest neighbor interactions is applied to study compaction processes in granular media. An equivalent particle-hole picture is introduced, with the holes being associated to the domain walls of the Ising model. Trying to mimic the experiments, a series of taps separated by large enough waiting times, for which the system freely relaxes, is considered. The free relaxation of the system corresponds to a $T=0$ dynamics which can be analytically solved. There is an extensive number of metastable states, characterized by all the holes being isolated. In the limit of weak tapping, an effective dynamics connecting the metastable states is obtained. The steady state of this dynamics is analyzed, and the probability distribution function is shown to have the canonical form. Then, the stationary state is described by Edwards thermodynamic granular theory. Spatial correlation functions in the steady state are also studied.
\end{abstract}

DOI: 10.1103/PhysRevE.66.041308

PACS number(s): 81.05.Rm, 05.50.+q, 45.70.Cc

\section{INTRODUCTION}

Granular systems have attracted the attention of physicists in recent years. A review of some features of granular matter can be found in Refs. [1,2]. One of the most outstanding problems is the phenomenon of compaction, i.e., the increase of the density of a loosely packed granular system when submitted to vibration or, more generally, to some kind of external excitation. Compaction has been extensively analyzed in a series of experiments by the Chicago group [3-5]. Starting from a low-density configuration, near the random loose packed state, a system of monodisperse glass beads was vertically tapped. Between taps, a long enough time was considered, so that the system reached a mechanically stable (metastable) configuration before the next tap started. The density was measured in the metastable states, and its evolution as a function of the number of taps was studied. The parameter controlling the dynamics of the system is the dimensionless vibration intensity $\Gamma=a / g$, where $a$ is the peak acceleration in the tap, and $g$ is the gravity. The density was observed to increase very slowly towards a steady value following an inverse logarithmic law $[3,4]$, the steady density being a monotonic decreasing function of the vibration intensity $[5,6]$. Several models, with different underlying physical mechanisms, have been proposed to understand this behavior [7-12], but a complete and detailed physical theory is still lacking.

Simple Ising systems are often used as a first approximation to many different phenomena in statistical physics. In a recent work $[13,14]$, the one-dimensional Ising model with nearest neighbor interactions has been applied to analyze the problem of compaction in dense granular media. This system can be regarded as one of the simplest cases of spin models on random graphs, which have been very recently used to investigate different aspects of granular matter $[11,15,16]$. The "tapping" process is simulated in the following way, in order to mimic what is done in the experiments [3-5]. First,

\footnotetext{
*Electronic address: prados@us.es

${ }^{\dagger}$ Electronic address: brey@us.es
}

the system freely relaxes until it gets stuck in a metastable configuration $(n=0)$. This is done by considering a modified Glauber dynamics at $T=0$, in which only those transitions lowering the energy of the system are permitted. Thus, all the configurations with no spins antiparallel to both of their nearest neighbors are metastable, i.e., they are absorbent states [17] of this $T=0$ dynamics. Starting from the metastable state $n=0$, the system is "vibrated" by allowing each spin to flip with probability $p$, independently of the state of its neighbors. Afterwards, another free relaxation at $T=0$ is considered, and the system reaches a new metastable configuration $n=1$. By repeating this process, a chain $n$ $=0,1,2, \ldots$ of metastable configurations is generated. It is found that the energy of the system is a monotonic decreasing function of the number of taps $n$. This Ising model can be mapped on a particle-hole model, in which a particles is associated to site $i$ if spins $i$ and $i+1$ are parallel, and a hole is spins $i$ and $i+1$ are antiparallel. In this way, holes correspond to the domain walls between arrays of parallel spins. Interestingly, the dynamics at $T=0$ can be analytically solved in the particle-hole picture [18]. As a decrease in the energy corresponds to a decrease in the number of domain walls, the density of holes is a monotonic decreasing function of the number of taps $n$ in the tapping process, i.e., compaction takes place. In the reminder of the paper, we will refer to this system as the TIM (tapped Ising model).

An analogous description of tapping processes was previously introduced for the one-dimensional one-spin facilitated Ising model (1SFM) [10,19], originally proposed by Fredrickson and Andersen in the context of structural relaxation in glassy systems [20]. In this model there are no interactions, but only an applied external field $h$, and a spin can only flip if at least one of its nearest neighbors is in the excited state. This system is also equivalent to a particle-hole model, in which particles are associated to the spins aligned with the field, while holes correspond to the spins in the excited state. Then, facilitated spin flips are equivalent to adsorption and desorption of particles on the onedimensional lattice. These processes can only occur provided that there is a hole on at least one of the nearest neighbor sites of the flipping spin. The tapping process is modeled in 
the following way [10]. First, the system relaxes at $T=0$, where only adsorption processes are allowed, until the system reaches a metastable configuration $n=0$. Due to the facilitation rule, the metastable states are characterized by all the holes being isolated, i.e., surrounded by two particles. Then, starting from the metastable state $n=0$, vibration is introduced by letting the system evolve, with both facilitated adsorption and desorption, for a given time $t_{0}$. Afterwards, the system relaxes again at $T=0$, which leads the system to a new metastable state $n=1$. Iteration of this process gives a set $n=0,1,2, \cdots$ of metastable configurations, with the density of particles being an increasing function of the number of taps $n[10,19]$.

One of the most interesting physical questions in the problem of compaction is the description of the steady state reached by the system in the limit of an infinite number of taps. Note that thermal energy is irrelevant for granular systems. The important energy scale for a grain of mass $m$ and diameter $d$ is $m g d$, where $g$ is the gravity. In a typical granular system, $m g d / k_{B} T \simeq 10^{12}$ at room temperature. Therefore, while molecular systems explore phase space due to thermal fluctuations, in a powder thermal energy is negligible. Unless the system is externally perturbed, each metastable configuration would last indefinitely. Thus, thermodynamics is not directly applicable to powders. Nevertheless, some years ago, Edwards and co-workers [21,22] made the hypothesis that the steady state of an externally perturbed granular system can be described by an extension of the usual statistical mechanics concepts to granular media. The central point is the ergodic hypothesis for externally perturbed powders: in the steady state, all the metastable configurations of a granular assembly occupying the same volume are equiprobable. Besides, its most stable configuration corresponds to the minimum volume. Therefore, the volume of a granular system is the analog to the energy for a molecular system. The entropy is defined as the logarithm of the number of metastable configurations, which is expected to be an extensive quantity. Then, it is possible to define a new parameter, the compactivity $X=\partial V / \partial S$, playing the role of the temperature in a molecular system, with the limit $X=0$ giving the most compact state.

In the last years, a lot of effort have been carried out in order to understand if the above "equilibrium statistical mechanics" or "thermodynamic" approach describes accurately the steady state of an externally perturbed granular system. Most of this effort has been focused on the analysis of simple models [13,14,19,23-27], although there has been also some attempts to test Edwards' theory in experiments with real granular systems [5]. Very recently, the theory has been checked in a numerical experiment with a realistic granular matter model, specially conceived to be reproducible in the laboratory [28]. In the context of simple models, there is some numerical evidence of the validity of the thermodynamic description in the limit of gently tapped systems [13,24-27], although for stronger tapping the situation is not clear. In fact, numerical results [24,27] show that in the limit of strong tapping, the Edwards measure does not provide an accurate description of the stationary state, at least in some spin models. This has suggested an extension of the Edwards approach by introducing a "restricted" measure [24,27]. On the other hand, analytical results are scarce, even for the simplest models. To the best of our knowledge, the only system in which Edwards theory has been analytically derived for tapping dynamics is the 1SFM described above, in the limit of weak tapping [19]. An effective dynamics for the tapping process, connecting metastable configurations, was obtained and the steady probability distribution was shown to have the canonical form. This leads to a relationship between Edwards' compactivity and the dimensionless vibration intensity. Let us also mention that Crisanti et al. [29] have studied one-dimensional kinetically restricted models to address the validity of the Stillinger-Weber construction, an approach that is related to the Edwards measure.

Due to the lack of analytical results, it seems interesting to investigate the possibility of deriving a thermodynamical description in the steady state of other simple models. This is an important task from a theoretical point of view. First, it is a relevant question if the thermodynamic picture is valid or not for models reproducing the experimentally observed behavior in granular systems. Second, if the answer is positive, it might be possible to derive relationships between the parameters controlling the evolution of the system, for instance, the tapping intensity $\Gamma$ in the compaction experiment, and the compactivity $X$, which characterizes the stationary state. In this paper, we will center on the analysis of the effective dynamics and the steady state of the TIM, also in the limit of a gently tapped system. We will obtain the effective dynamics between metastable states as an analytical approximation to the original tapping dynamics. This will allow us to derive, also analytically, the steady state probability distribution.

The paper is organized as follows. In Sec. II the model is introduced, while the analytical solution of the modified Glauber dynamics at $T=0$ is presented in Sec. III. Section IV is devoted to the derivation of the effective dynamics in the limit of weak tapping. The properties of the steady state reached in the limit of an infinite number of taps are discussed in Sec. V. It is shown that the steady probability can be written in the canonical form, with the role of the energy played by the volume and the temperature being substituted by a new parameter, the compactivity, which is related to the tapping intensity. Then, the steady state follows the statistical mechanics theory of Edwards and coworkers [21,22]. Finally, Sec. VI contains a summary of the work and some final remarks.

\section{AN ISING MODEL AT $T=0$ (FREE RELAXATION)}

The Hamiltonian of the one-dimensional Ising model is given by

$$
\mathcal{H}=-J \sum_{i=1}^{N} \sigma_{i} \sigma_{i+1},
$$

where $J>0$ is the ferromagnetic coupling constant, $N$ is the number of spins on the lattice, and $\sigma_{i}= \pm 1$ is the spin variable at site $i$. We will consider periodic boundary conditions, so that formally $\sigma_{N+1}=\sigma_{1}$. The time evolution of the sys- 
tem is governed by single-spin-flip dynamics [30]. The probability $p(\boldsymbol{\sigma}, t)$ for finding the system in configuration $\boldsymbol{\sigma}$ $\equiv\left\{\sigma_{i}\right\}$ at time $t$ obeys a master equation of the form

$$
\partial_{t} p(\boldsymbol{\sigma}, t)=\sum_{i=1}^{N}\left[w\left(\boldsymbol{\sigma} \mid R_{i} \boldsymbol{\sigma}\right) p\left(R_{i} \boldsymbol{\sigma}, t\right)-w\left(R_{i} \boldsymbol{\sigma} \mid \boldsymbol{\sigma}\right) p(\boldsymbol{\sigma}, t)\right] .
$$

Here $R_{i} \boldsymbol{\sigma}$ is the configuration obtained from $\boldsymbol{\sigma}$ by just changing the state of spin $i$, and $w\left(\boldsymbol{\sigma} \mid \boldsymbol{\sigma}^{\prime}\right)$ stands for the transition rate from configuration $\boldsymbol{\sigma}^{\prime}$ to $\boldsymbol{\sigma}$. Following Lefevre and Dean $[13,14]$, we introduce a $T=0$ dynamics such that only those spin flips decreasing the energy of the system are allowed. Namely, the transition rates are

$$
w\left(R_{i} \boldsymbol{\sigma} \mid \boldsymbol{\sigma}\right)=\frac{\alpha}{4}\left(1-\sigma_{i-1} \sigma_{i}\right)\left(1-\sigma_{i} \sigma_{i+1}\right)
$$

It is easily verified that the above expression vanishes unless spin $i$ is antiparallel to both of its nearest neighbors. The constant $\alpha$ defines the basic time scale of the system. Interestingly, the most general transition rates bringing the Ising model to equilibrium at temperature $T$ are [30]

$$
\begin{aligned}
w\left(R_{i} \boldsymbol{\sigma} \mid \boldsymbol{\sigma}\right)= & \frac{\alpha}{4}\left[1+\delta \sigma_{i-1} \sigma_{i+1}-\frac{1+\delta}{2}\right. \\
& \left.\times \sigma_{i}\left(\sigma_{i-1}+\sigma_{i+1}\right) \tanh \left(\frac{2 J}{k_{B} T}\right)\right],
\end{aligned}
$$

where $\delta$ is an arbitrary constant. The usual Glauber dynamics corresponds to the choice $\delta=0$, whereas Eq. (2.3) is the zero temperature limit of the case $\delta=1$. The dynamics defined by the transition rates (2.3) cannot be solved in the standard way [30], since the hierarchy of equations for the moments $C_{n}=\left\langle\sigma_{i} \sigma_{i+1}\right\rangle$ is not closed.

Let us go to an equivalent description of the Ising model in terms of particles and holes, by introducing a new set of variables

$$
m_{i}=\frac{1-\sigma_{i} \sigma_{i+1}}{2} .
$$

If spins at sites $i$ and $i+1$ are antiparallel, it is $m_{i}=1$ and we will refer to the site $i$ as empty or, equivalently, as being occupied by a hole. On the other hand, if spins at site $i$ and $i+1$ are parallel it is $m_{i}=0$, and site $i$ is occupied by a particle. Therefore, holes are associated to the boundaries between domains of parallel spins, i.e., to the so-called domain walls of the system. Due to the periodic boundary conditions, the number of holes must be even in any configuration.

In terms of the $m_{i}$ variables, the Hamiltonian (2.1) reads

$$
\mathcal{H}(\boldsymbol{m})=-J \sum_{i=1}^{N}\left(1-2 m_{i}\right)=-J N+2 J \sum_{i=1}^{N} m_{i}
$$

A dimensionless energy per spin $\varepsilon$ can be defined as

$$
\varepsilon=\frac{\langle\mathcal{H}\rangle_{t}}{J N}=-1+\frac{2}{N} \sum_{i=1}^{N}\left\langle m_{i}\right\rangle_{t}
$$

where we use the notation

$$
\langle A(\boldsymbol{m})\rangle_{t}=\sum_{\boldsymbol{m}} A(\boldsymbol{m}) p(\boldsymbol{m}, t),
$$

for an arbitrary function $A(\boldsymbol{m})$ of the site variables $m_{i}$.

In the hole-particle description of the dynamics, the flip of spin $i$ modifies both the values of $m_{i-1}$ and $m_{i}$. Then, from Eq. (2.3) we get for the transition rates in the $\boldsymbol{m}$ variables

$$
w\left(R_{i-1} R_{i} \boldsymbol{m} \mid \boldsymbol{m}\right)=\alpha m_{i-1} m_{i},
$$

where $R_{i}$ is now the operator which transforms $m_{i}$ into 1 $-m_{i}$. The master equation for the probability $p(\boldsymbol{m}, t)$ is

$$
\begin{aligned}
\partial_{t} p(\boldsymbol{m}, t)= & \sum_{i}\left[w\left(\boldsymbol{m} \mid R_{i-1} R_{i} \boldsymbol{m}\right) p\left(R_{i-1} R_{i} \boldsymbol{m}, t\right)\right. \\
& \left.-w\left(R_{i-1} R_{i} \boldsymbol{m} \mid \boldsymbol{m}\right) p(\boldsymbol{m}, t)\right] .
\end{aligned}
$$

In the dynamics defined by Eqs. (2.9) and (2.10), the only possible transitions are the simultaneous adsorption of two particles on any two neighboring empty sites. After a long enough time, the system becomes trapped in a metastable state characterized by all the holes being isolated, i.e., all the empty sites surrounded by two particles. Of course, the particular metastable state reached by the system will depend on the initial conditions.

The present model displays some similarities as compared with the 1SFM [20] at $T=0$. In the latter, a particle can be adsorbed on an empty site as long as at least one of its nearest neighboring sites is empty $[10,19]$. Although the dynamics of both models are not equivalent, the metastable states are the same, being characterized by having all the empty sites isolated.

\section{ANALYTICAL SOLUTION OF THE DYNAMICS AT $T=0$}

Let us define the set of moments

$$
D_{r}(t)=\left\langle m_{k} m_{k+1} \cdots m_{k+r}\right\rangle_{t},
$$

with $r \geqslant 0$. In the following, we will restrict ourselves to homogeneous states, so that $D_{r}(t)$ does not depend on the position $k$ of the first site considered. The lowest moment

$$
D_{0}(t)=\left\langle m_{k}\right\rangle_{t}
$$

is the density of holes. This quantity can be related to the energy per particle by means of Eq. (2.7),

$$
\varepsilon=-1+2 D_{0} .
$$

A hierarchy of equations for the moments $D_{r}(t)$ is easily obtained from the master equation (2.10),

$$
\partial_{t} D_{r}(t)=-\alpha r D_{r}(t)-2 \alpha D_{r+1}(t),
$$


valid for all $r \geqslant 0$. In order to solve the above hierarchy we introduce the generating function

$$
G(x, t)=\sum_{r=0}^{\infty} \frac{x^{r}}{r !} D_{r}(t),
$$

from which all the moments $D_{r}(t)$ can be obtained through

$$
D_{r}(t)=\left[\frac{\partial^{r} G(x, t)}{\partial x^{r}}\right]_{x=0} .
$$

From Eq. (3.4), it follows that the function $G(x, t)$ obeys the first-order partial differential equation

$$
\partial_{t} G(x, t)+\alpha(x+2) \partial_{x} G(x, t)=0 .
$$

By using standard techniques, the general solution of the above equation is found to be

$$
G(x, t)=G_{0}\left[(x+2) e^{-\alpha t}-2\right],
$$

where the function $G_{0}(y)$ is the initial condition, i.e.,

$$
G_{0}(y) \equiv G(y, 0)=\sum_{r=0}^{\infty} \frac{y^{r}}{r !} D_{r}(0) .
$$

In the long time limit it is

$$
G(x, \infty)=G_{0}(-2),
$$

so that

$$
\lim _{t \rightarrow \infty} D_{0}(t)=G_{0}(-2)
$$

and

$$
\lim _{t \rightarrow \infty} D_{r}(t)=0
$$

for $r \geqslant 1$. The last result shows that all the holes become isolated in the long time limit, and the probability of finding $r+1$ consecutive holes, which equals $D_{r}$, vanishes for $r$ $\geqslant 1$. The asymptotic density of holes depends on the initial state, as indicated by Eq. (3.11). In fact, it is trivially seen that the hierarchy of Eqs. (3.4) admits as a solution any constant value of $D_{0}$ as long as $D_{r}=0$ for $r \geqslant 1$.

Now, let us specify the initial condition. We will consider that the system is in equilibrium at temperature $T$ at $t=0$. The equilibrium distribution is given by the canonical distribution corresponding to the Hamiltonian (2.1),

$$
p_{T}^{\mathrm{eq}}(\boldsymbol{\sigma})=\frac{e^{-\beta \mathcal{H}(\boldsymbol{\sigma})}}{\sum_{\boldsymbol{\sigma}} e^{-\beta \mathcal{H}(\boldsymbol{\sigma})}},
$$

or, equivalently,

$$
p_{T}^{\mathrm{eq}}(\boldsymbol{m})=\frac{e^{-\beta \mathcal{H}(\boldsymbol{m})}}{\sum_{\boldsymbol{m}} e^{-\beta \mathcal{H}(\boldsymbol{m})}}
$$

where $\beta=\left(k_{B} T\right)^{-1}$. Using the Hamiltonian (2.6), it is easy to show that

$$
\begin{aligned}
& p_{T}^{\mathrm{eq}}(\boldsymbol{m})=\prod_{i=1}^{N} p_{T}^{\mathrm{eq}}\left(m_{i}\right), \\
& p_{T}^{\mathrm{eq}}\left(m_{i}\right)=\frac{e^{-2 \beta J m_{i}}}{1+e^{-2 \beta J}} .
\end{aligned}
$$

Therefore,

$$
\begin{gathered}
D_{0, T}^{\mathrm{eq}}=\left\langle m_{k} ; T\right\rangle_{\mathrm{eq}}=\frac{e^{-2 \beta J}}{1+e^{-2 \beta J}}=a, \\
D_{r, T}^{\mathrm{eq}}=\left\langle m_{k} m_{k+1} \cdots m_{k+r} ; T\right\rangle_{\mathrm{eq}}=\left\langle m_{k} ; T\right\rangle_{\mathrm{eq}}^{r+1}=a^{r+1},
\end{gathered}
$$

where $0 \leqslant a \leqslant 1$. The value $a=0$ corresponds to $\beta \rightarrow \infty$ (T $\left.\rightarrow 0^{+}\right)$, and $a=1$ to $\beta \rightarrow-\infty\left(T \rightarrow 0^{-}\right)$. In the limit $\beta \rightarrow 0$ $(T \rightarrow \infty)$, it is $a=1 / 2$, which corresponds to the most disordered state.

Therefore, the initial condition corresponding to an equilibrium state is given by

$$
D_{r}(0)=a^{r+1}
$$

which leads to

$$
G_{0}(x) \equiv G(x, 0)=\sum_{r=0}^{\infty} \frac{x^{r}}{r !} a^{r+1}=a e^{a x} .
$$

With this choice, the solution given by Eq. (3.8) takes the form

$$
G(x, t)=a \exp \left\{a\left[(x+2) e^{-\alpha t}-2\right]\right\}
$$

and, consequently, by using Eq. (3.6),

$$
D_{r}(t)=a^{r+1} \exp \left[-\alpha r t+2 a\left(e^{-\alpha t}-1\right)\right] .
$$

As pointed out above, all the moments $D_{r}$ with $r \geqslant 1$ vanish in the long time limit, while the asymptotic value of the density of holes reads

$$
\lim _{t \rightarrow \infty} D_{0}(t)=a e^{-2 a}
$$

which depends on the initial density of holes $a$, being always smaller than it, since only adsorption processes are allowed in the $T=0$ dynamics. The dimensionless energy per particle in the metastable final configuration $\varepsilon_{\infty}$ follows directly from Eqs. (3.3) and (3.21),

$$
\varepsilon_{\infty}=-1+2 a e^{-2 a} .
$$


This expression agrees with the result obtained by Lefevre and Dean $[13,14]$. The energy $\varepsilon_{\infty}$ is maximum for $a=1 / 2$, i.e., when the system starts from a fully random configuration. Of course, this is equivalent to say that $D_{0}(\infty)$ has a maximum for $a=1 / 2$. The existence of this maximum is in contrast with the result for the 1SFM, where the asymptotic density of holes is a monotonic function of the initial density $[10,31]$.

Therefore, at $T=0$ the following picture emerges. Starting from any configuration, the system evolves until all the holes become isolated, i.e., it gets stuck in a metastable state characterized by all the moments $D_{r}$ vanishing for $r \geqslant 1$. Going back to the spin description, the metastable states are those such there is no spin antiparallel to both of its nearest neighbors. In other words, all the domains of parallel spins have, at least, a length of two sites. The density of holes in the metastable state, or the density of domain walls in the spin image, depends on the initial configuration, being given by Eq. (3.21).

\section{EFFECTIVE DYNAMICS FOR TAPPING PROCESSES}

Let us consider the model introduced in Sec. II to get a theoretical approach to the compaction processes in vibrated granular systems. The model is tapped in the following way $[13,14]$, trying to mimic what is done in the experiments with real granular materials [3-5]. First, the system freely relaxes as described in the preceding section, until getting trapped in a metastable configuration $(n=0)$, characterized by the absence of spins being antiparallel to both of their nearest neighbors. These configurations are absorbent states [17] for the dynamics at $T=0$. Second, starting from the metastable configuration $n=0$, the system is "vibrated." Each spin can flip with a probability $p$, independently of the state of its neighbors. Afterwards, another free relaxation at $T=0$ follows, until the system becomes again stuck in a new metastable configuration, $n=1$. By repeating this process, a chain of metastable configurations $n=0,1,2, \ldots$ is generated. It is important to note that we can restrict ourselves to values of $p$ in the interval $0 \leqslant p \leqslant 1 / 2$, since the same evolution of the energy is obtained for both $p$ and $1-p$. This is because a probability $1-p$ is equivalent to a simultaneous flip of all the spins (which does not change the energy), followed by a flip of each spin with probability $p$. Making use of the notation introduced in the introduction, we will refer to this tapped one-dimensional Ising model as the TIM.

If $p \ll 1$, the evolution of the system is very slow, since it is very improbable that a given spin flips. The dynamics will be dominated by those transitions in which only a few spins change their state during a vibration process. Therefore, for $p \ll 1$ an expansion in powers of $p$ may be useful, since we hope that retaining the lowest orders would provide a good approximation.

In the preceding section we have shown that, in the description of particles and holes, the metastable states are characterized by having all the holes isolated, i.e., there are no domains of parallel spins with length $l<2$. We are going to consider the evolution of the system in a single tap, defined as the sequence of a vibration process followed by a
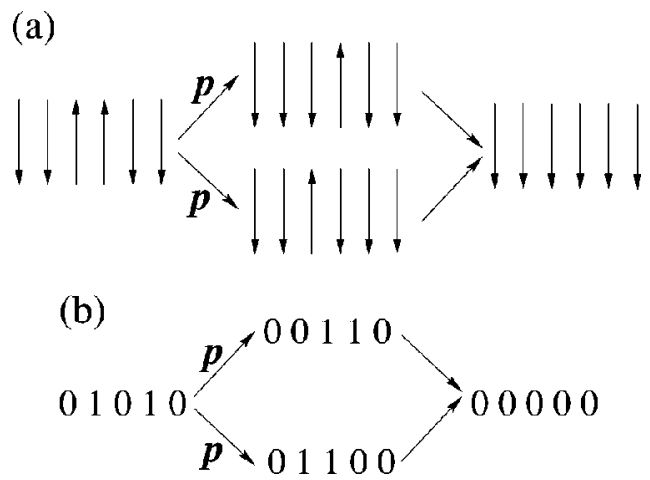

FIG. 1. (a) Destruction of a domain of length $l=2$ in the tapping process. In the vibration, one of the spins of the domain flips with probability $p$, and the remaining unstable domain of length $l=1$ disappears in the free relaxation at $T=0$. (b) The same trajectories, in the particle and hole picture.

free relaxation to a metastable configuration, to the lowest order in $p$. In this limit, only one flip during the vibration takes place. The analysis to be presented depends on the length $l$ of the domain of parallel spins containing the flipping spin. Our goal is to obtain expressions for the transition probabilities $W_{\mathrm{ef}}\left(\boldsymbol{m}^{\prime} \mid \boldsymbol{m}\right)$ from the initial metastable configuration $\boldsymbol{m}$ to the final one $\boldsymbol{m}^{\prime}$ in a single tap.

Let us assume first that the flipping spin belongs to a domain of initial length $l=2$, i.e., the transition is like

$$
\cdots \downarrow \downarrow \uparrow \uparrow \uparrow \downarrow \downarrow \ldots \rightarrow \ldots \downarrow \downarrow \underline{-} \uparrow \uparrow \downarrow \downarrow \ldots,
$$

where the flipping spin has been underlined. The probability that this process occurs in the cluster above is $p(1-p)^{5}$. Afterwards, in the free relaxation at $T=0$ the up-spin has to flip necessarily, reaching a new metastable state in which the domain with $l=2$ has disappeared. Therefore, to first order in $p$

$$
W_{\text {ef }}(\downarrow \downarrow \downarrow \downarrow \downarrow \downarrow \mid \downarrow \downarrow \uparrow \uparrow \downarrow \downarrow)=2 p,
$$

where the factor of 2 follows because of the other path connecting the same initial and final states, and corresponding to the flip of the spin on the right of the domain with $l=2$ during the vibration. These trajectories are shown in Fig. 1, both in the spin and in the particle-hole pictures. Introducing an usual notation, in the transition rates $W_{\text {ef }}\left(\boldsymbol{m}^{\prime} \mid \boldsymbol{m}\right)$ we have only indicated the sites involved in the given rearrangement. In the particle and hole picture, it is

$$
W_{\text {ef }}(00000 \mid 01010)=2 p,
$$

the process involves the elimination of both holes. During the vibration, one hole diffuses next to the other one, so that in the free relaxation two particles are simultaneously adsorbed on them, as it is shown in Fig. 1.

When the flipping spin belongs to a domain of length $l$ $>2$, we have to analyze two cases separately, by distinguishing whether the flipping spin during the vibration is located at the domain wall or not. In the former case, for instance 


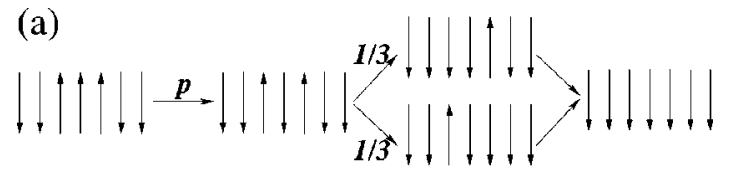

(b)

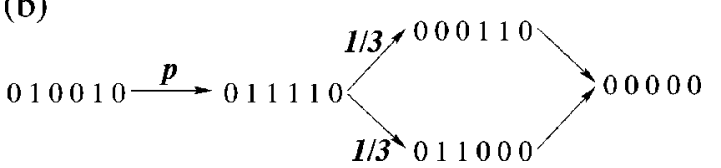

FIG. 2. (a) Trajectories leading to the destruction of a domain of length $l=3$ in a tapping process. In the vibration, the central spin flips with probability $p$. Afterwards, the system freely relaxes at $T$ $=0$. Paths leading to a final state identical to the initial one are not shown. (b) The same trajectories as in (a), in the particle-hole picture. Note that the flip of one spin corresponds to the change of two consecutive sites in the associated particle lattice.

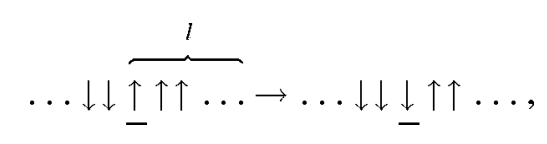

no transition occurs in the following free relaxation, since the final state in the vibration process is metastable. Therefore, the effective transition probability between the metastable states is

$$
W_{\mathrm{ef}}(\downarrow \downarrow \downarrow \uparrow \uparrow \mid \downarrow \downarrow \uparrow \uparrow \uparrow)=p
$$

Similarly,

$$
W_{\text {ef }}(\uparrow \uparrow \downarrow \downarrow \downarrow \mid \uparrow \uparrow \uparrow \downarrow \downarrow)=p
$$

These transitions are one-site diffusion processes of a hole in both directions,

$$
W_{\text {ef }}(0010 \mid 0100)=W_{\text {ef }}(0100 \mid 0010)=p,
$$

which are present for all $l>2$.

When one of the internal spin flips, we have to analyze separately $l=3$ and $l \geqslant 4$. First, let us consider $l=3$. The internal spin is a nearest neighbor of both the spins at the domain walls, and the rearrangement occurring in the vibration has the form

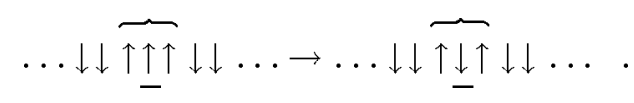

Afterwards, in the free relaxation, any of the three spins of the domain can flip, with the same probability, i.e., 1/3. If it is the central spin the one flipping, returning then to its original state, nothing has occurred globally, and the group is again in the initial state. On the other hand, if any of the external spins of the domain flips first, say the one on the left, the reached state is not metastable yet. Then, the free relaxation involves another flip, in which the upward spin has necessarily to go down. Therefore, the effective transition probability for the complete process is

$$
W_{\text {ef }}(\downarrow \downarrow \downarrow \downarrow \downarrow \downarrow \downarrow \mid \downarrow \downarrow \uparrow \uparrow \uparrow \downarrow \downarrow)=\frac{2}{3} p .
$$

TABLE I. Probabilities of the first-order transitions in a single tap, connecting metastable states.

\begin{tabular}{lccc}
\hline \hline \multicolumn{1}{c}{ Process } & Initial state & Final state & $W_{\text {ef }}$ \\
\hline One-site diffusion & 0100 & 0010 & $p$ \\
& 0010 & 0100 & $p$ \\
Two-sites diffusion & 01000 & 00010 & $p$ \\
& 00010 & 01000 & $p$ \\
Destruction of a hole pair & 01010 & 00000 & $2 p$ \\
& 010010 & 000000 & $\frac{2}{3} p$ \\
\hline \hline
\end{tabular}

The trajectories leading to this rearrangement are shown in Fig. 2. The factor of $2 / 3$ appears because the final state is the same, independently of which is the first external spin flipping in the free relaxation, and each trajectory in Fig. 2 contributes $p / 3$. In the particle-hole picture, the process consists of the occupation of two holes separated by two particles, with a probability

$$
W_{\mathrm{ef}}(000000 \mid 010010)=\frac{2}{3} p .
$$

In a domain of length $l \geqslant 4$, there are $l-2$ internal spins. Suppose that the one next to the left wall flips in the vibration,

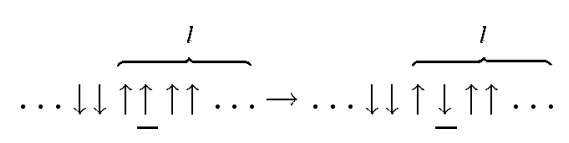

To the right of the flipping spin, a stable domain of length $l-2$ appears, and the spin to its left must move downwards in the free relaxation, i.e., the domain wall moves two sites to the right in the whole process. The probability of the transition is

$$
W_{\text {ef }}(\downarrow \downarrow \downarrow \downarrow \uparrow \uparrow \mid \downarrow \downarrow \uparrow \uparrow \uparrow \uparrow)=p,
$$

or, in the particle-hole description,

$$
W_{\mathrm{ef}}(00010 \mid 01000)=p \text {. }
$$

The process corresponds to a two-sites diffusion of the hole to the right. Obviously, it is also possible that a hole diffuses two sites to the left, corresponding to the flip of the internal spin next to the right wall in the vibration,

$$
W_{\mathrm{ef}}(01000 \mid 00010)=p \text {. }
$$

Finally, if the internal spin which flips in the vibration is not next to any of the domain walls, it has to return to its original state in the free relaxation, and there is no global transition in the tap. The obtained transition probabilities up to first order in $p$ are summarized in Table I. The particle-hole description is used, since it is more convenient for the analysis of the compaction process. Therefore, to the lowest order, with only one flip during the vibration, the density of particles cannot decrease and compaction takes place. When higher orders are retained, processes leading to a decrease of the number of particles show up, as it will be discussed below. 
(a)

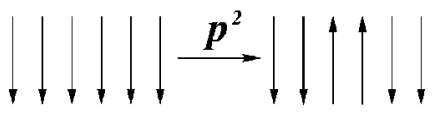

(b)

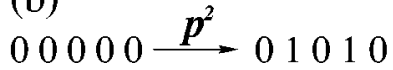

FIG. 3. (a) Creation of a domain of length $l=2$ in the tapping process. In the vibration, two consecutive spins belonging to a domain of length $l \geqslant 6$ flip with probability $p^{2}$. As a consequence, a new stable domain of length $l=2$ shows up. (b) The preceding trajectory, in the particle-hole picture.

The description of the effective dynamics between metastable states is not complete, even qualitatively, if it is restricted to the lowest order in $p$. In particular, the existence of a steady state characterized by the flipping probability in the vibration $p[13,14]$ is lost. The Markov process describing the dynamics between metastable states is not irreducible, and the configuration with all the sites being occupied by particles is an absorbent state [17] of the dynamics. Therefore, in order to have a more complete description, we are led to consider higher orders in $p$, i.e., processes involving more than one transition during the vibration. This will be done in a physical way, similar to that of Ref. [19]. We are not going to consider those second-order processes (two flips in the vibration) whose effect can be obtained by means of a combination of two processes of order $p$, but only those processes for which the effective transition probability $W_{\text {ef }}$ vanishes to the lowest order. In particular, this is the case for all those trajectories decreasing the density of particles, as already mentioned. The inclusion of these processes modifies essentially the physics of the tapping process, so that they must be taken into account in our effective dynamics.

Then, we will consider that there are two flips take place in the vibration. As in the free relaxation only transitions decreasing the energy are allowed, we have to analyze two cases: (i) flip of two nearest neighbors spins, and (ii) flip of two spins separated by one site. If the two flipping spins are separated by more than one site, the local free relaxations associated to each of them are independent, and the result is a product of two first-order transitions.

Suppose the transition during the vibration in the cluster depicted in Fig. 3, whose probability is $p^{2}(1-p)^{4}$. In the free relaxation no transition can happen, since a metastable domain of length $l=2$ has been created. Therefore, to the lowest order it is

$$
W_{\text {eff }}(\downarrow \downarrow \uparrow \uparrow \downarrow \downarrow \mid \downarrow \downarrow \downarrow \downarrow \downarrow \downarrow)=p^{2},
$$

or, in the particle-hole picture,

$$
W_{\text {ef }}(01010 \mid 00000)=p^{2} .
$$

In order to derive this transition probability it has been assumed that the domain which the flipping spins belong to initially is at least of length $l=6$. If it has a smaller length, it is easily shown that the resulting rearrangement can be obtained as a combination of two first-order processes. The (a)

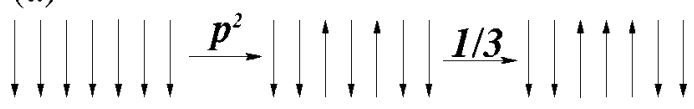

(b)

$000000 \stackrel{p^{2}}{\longrightarrow} 011110 \stackrel{1 / 3}{\longrightarrow} 010010$

FIG. 4. (a) In the vibration, two spins of the domain of length $l \geqslant 7$ flip with probability $p^{2}$, creating three consecutive unstable domains of length $l=1$. If, in the subsequent free relaxation, it is the central spin the one which flips, a new stable domain of length $l=3$ appears. (b) The same trajectory, shown for particles and holes.

other kind of second-order processes, we have to include in our approximation corresponds to a transition during the vibration of the form

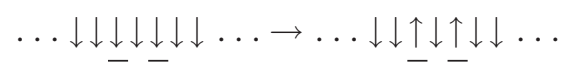

whose probability, to leading order, is $p^{2}$. In the free relaxation process, first either one of the underlined spins which have just flipped or the one between them flips, the three changes having the same probability. In the former case, the group of spins returns to the initial configuration, all of them upwards. On the other hand, if the central spin flips, a new domain of length $l=3$ appears, with probability

$$
W_{\text {ef }}(\downarrow \downarrow \uparrow \uparrow \uparrow \downarrow \downarrow \mid \downarrow \downarrow \downarrow \downarrow \downarrow \downarrow \downarrow)=\frac{1}{3} p^{2} .
$$

The trajectory leading to this transition is shown in Fig. 4. In terms of particles and holes

$$
W_{\text {ef }}(010010 \mid 000000)=\frac{1}{3} p^{2} .
$$

It is easy to convince oneself that there are no more secondorder transitions increasing the number of holes, and that any other second-order process can be decomposed in a product of first-order transitions. The new transitions appearing to second-order are shown in Table II. Together with the firstorder processes in Table I, they define the approximate effective model for tapping processes we are going to analyze in the following. It is important to note that the introduction of the second-order processes is fundamental from a physical point of view. The processes described in Figs. 3 and 4, with probabilities given by Eqs. (4.16) and (4.19), are the inverse of the processes in Figs. 1 and 2, Eqs. (4.3) and (4.10), respectively. Therefore, the Markov process defined by the transition probabilities in Tables I and II is irreducible [17], i.e., all the states are connected by a chain of transitions with

TABLE II. Probabilities of the second-order processes between metastable states leading to an increase of the number of holes.

\begin{tabular}{cccc}
\hline \hline Process & Initial state & Final state & $W_{\mathrm{ef}}$ \\
\hline Creation of a hole pair & 00000 & 01010 & $p^{2}$ \\
& 000000 & 010010 & $\frac{1}{3} p^{2}$ \\
\hline
\end{tabular}


nonzero probability. This property will be fundamental in the analysis of the steady state, presented in the following section.

\section{STEADY STATE SOLUTION}

As pointed out above, the Markov process defined by the effective transition probabilities connecting metastable states is irreducible [17] and, consequently, there is a unique steady state for each given value of $p$. This steady state will be reached by the system from any initial configuration. Besides, it will be shown that the system described by the effective master equation verifies detailed balance. By using this property we will be able to obtain the steady distribution analytically. With regards to the original model, the expression holds in the limit of gently tapped systems, $p \ll 1$, for which the effective transition probabilities of Tables I and II have been obtained.

In order to calculate the steady distribution function, we will bet a priori on a stationary solution $P_{s}(\boldsymbol{m})$ of the master equation for the tapping process verifying the detailed balance condition,

$$
W_{\mathrm{ef}}\left(\boldsymbol{m} \mid \boldsymbol{m}^{\prime}\right) P_{s}\left(\boldsymbol{m}^{\prime}\right)=W_{\mathrm{ef}}\left(\boldsymbol{m}^{\prime} \mid \boldsymbol{m}\right) P_{s}(\boldsymbol{m})
$$

Given the uniqueness of the steady state, if a solution is found in this way, its own existence will be the proof of the detailed balance property in the system. Detailed balance implies that all the configurations $\boldsymbol{m}^{(k)}$ having the same number of holes $k$ will be equiprobable, since they are connected through diffusion processes, which are isotropic. Their probability will be denoted by $P_{s}\left(\boldsymbol{m}^{(k)}\right)$. Moreover, for the processes changing the density of the system it is

$$
\frac{P_{s}\left(\boldsymbol{m}^{\prime(k+2)}\right)}{P_{s}\left(\boldsymbol{m}^{(k)}\right)}=\frac{W_{\mathrm{ef}}\left(\boldsymbol{m}^{\prime(k+2)} \mid \boldsymbol{m}^{(k)}\right)}{W_{\mathrm{ef}}\left(\boldsymbol{m}^{(k)} \mid \boldsymbol{m}^{\prime(k+2)}\right)}=\frac{p}{2} .
$$

This expression applies for both pairs of transitions with nonzero probability, given by Eqs. (4.16) and (4.3), and Eqs. (4.19) and (4.10), respectively (see also Tables I and II). Consequently,

$$
P_{S}\left(\boldsymbol{m}^{(k)}\right)=\frac{1}{Z}\left(\frac{p}{2}\right)^{k / 2}
$$

where $Z$ is a normalization constant, and we have taken into account that the number of holes is always even. Defining a new variable $X$ by

$$
e^{-1 / X}=\sqrt{\frac{p}{2}}
$$

the steady probability distribution can be written in the "canonical" form

$$
P_{s}\left(\boldsymbol{m}^{(k)}\right)=\frac{1}{Z} e^{-k / X},
$$

so that $X$ is identified as the compactivity of Edwards' statistical mechanics theory of powders $[21,22]$. The number of holes $k$ plays the role of the volume or, more precisely, the excess volume from the densest state. The normalization constant $Z$ is the analog to the partition function. From it, all the steady properties of the system can be obtained in the standard way.

The calculation of $Z$ is quite an easy task,

$$
\begin{gathered}
Z=\sum_{k=0, k \text { even }}^{N / 2} Z_{k}, \\
Z_{k}=\Omega_{k}^{(N)} e^{-k / X},
\end{gathered}
$$

with $\Omega_{k}^{(N)}$ being the number of metastable states with $k$ holes for a lattice with $N$ sites. The maximum number of holes is $N / 2$ (we are assuming that $N$ is even), and the number of holes $k$ must be even in the TIM because of the periodic boundary conditions. A simple combinatorial argument leads to

$$
\Omega_{k}^{(N)}=\frac{N(N-k-1) !}{k !(N-2 k) !} .
$$

In the large $N$ limit, the sum in Eq. (5.6a) can be evaluated by the saddle point method, since $Z_{k}$ has a sharp maximum as a function of $k$, with the result

$$
\ln \zeta \equiv \frac{1}{N} \ln Z=\ln \frac{1+\left(1+4 e^{-1 / X}\right)^{1 / 2}}{2} .
$$

The number of holes is the property analogous to the energy of a molecular system, and the steady hole density reads

$$
D_{0}^{s}=-\frac{\partial \ln \zeta}{\partial(1 / X)}=\frac{\left(1+4 e^{-1 / X}\right)^{1 / 2}-1}{2\left(1+4 e^{-1 / X}\right)^{1 / 2}} .
$$

It must be stressed that $D_{0}^{s}=\bar{k} / N$, being $\bar{k}$ the value of the number of holes $k$ for which $Z_{k}$ reaches its maximum. The steady probability distribution is a very sharply peaked function around $\bar{k}$, which assures the equivalence of the microcanonical and canonical ensembles for the calculation of the mean values of the physical properties in the steady state. The stationary density of holes $\rho^{s}=1-D_{0}^{s}$ is a monotonic decreasing function of the compactivity $X$. As the compactivity, given by Eq. (5.4), increases with the vibration intensity $p, \rho^{s}$ is also a monotonic decreasing function of the vibration intensity, a behavior analogous to that of real granular systems $[5,6]$. In the limit $p \ll 1$, Eq. (5.9) reduces to

$$
D_{0}^{s} \sim e^{-1 / X}=\sqrt{p / 2} .
$$

In Refs. [13,14], it was found that the steady density of holes for arbitrary $p$ is given by the solution of the equation 


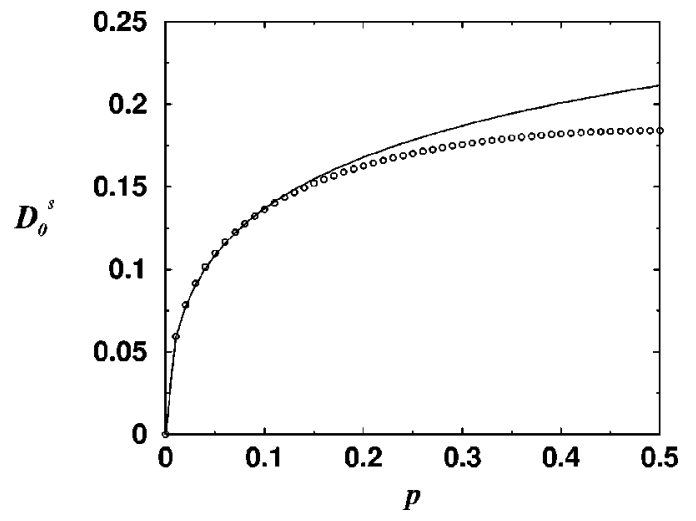

FIG. 5. Comparison of the numerical evaluation of the steady density of holes, as given by Eq. (5.11), (circles) and the analytical explicit expression obtained from the effective dynamics approach, Eq. (5.9). The agreement is quite good for $p \leqq 0.15$.

$$
D_{0}^{s}=\left[2 p q+D_{0}^{s}(1-4 p q)\right] \exp \left[-\frac{2 p q}{2 p q+D_{0}^{s}(1-4 p q)}\right],
$$

with $q=1-p$. Of course, as Eq. (5.11) gives the exact value of the density of holes, it is symmetric against the change $p \rightarrow 1-p$. In the limit $p \ll 1$, the above expression yields $D_{0}^{s} \sim \sqrt{p / 2}$, which agrees with the small $p$ limit of the expression obtained from the effective dynamics, Eq. (5.9). In Fig. 5 , we compare the density of holes as a function of $p$, obtained from Eqs. (5.9) and (5.11). It is observed that the agreement is quite good for $p \leqq 0.15$, i.e., for a steady density of particles $\rho^{s}=1-D_{0}^{s} \geq 0.85$. On the other hand, the leading behavior for $p \ll 1$, Eq. (5.10), only holds for very small values of $p, p \leq 10^{-3}$, as it is clearly shown in Fig. 6 . Therefore, the accuracy of the results obtained from the effective dynamics picture extends further than what might be expected from a second-order theory in the flipping probabil-

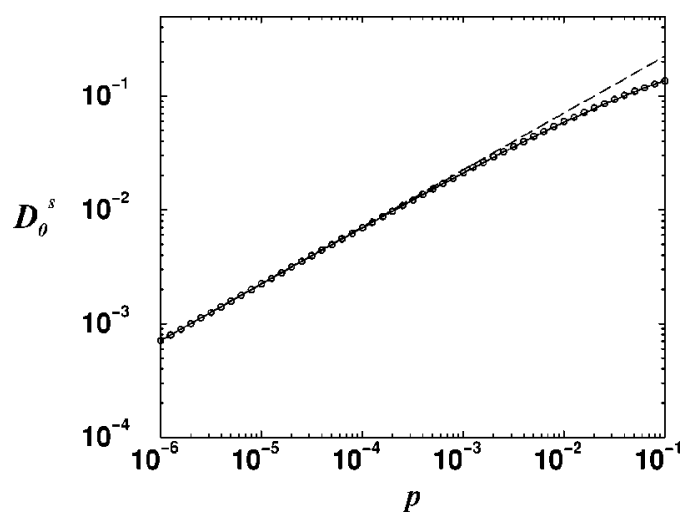

FIG. 6. Comparison of the numerical evaluation of the steady density of holes, as given by the exact solution Eq. (5.11) (circles), the analytical expression obtained from the effective dynamics approach, Eq. (5.9) (solid line), and their leading behavior (5.10) in the limit $p \ll 1$ (dashed line), in the interval $10^{-6}<p<10^{-1}$. It is observed that Eq. (5.10) only gives a good approximation in the very weak tapping regime, $p \lesssim 10^{-3}$, for which the steady density of particles $\rho^{s}=1-D_{0}^{s} \gtrsim 0.98$. ity $p$. In fact, this is not so surprising, because a similar behavior was found in the one-dimensional facilitated Ising model submitted to tapping processes [19]. The wide range of applicability presented by Eq. (5.9) can be understood on physical grounds, by realizing that the effective dynamics approach is not a standard second-order expansion in the flipping probability $p$. Among all the second-order processes, only those introducing new physically relevant transitions, i.e., transitions that cannot be written as a combination of first-order processes, are collected. This physically motivated expansion allows an analytical approach to the rather difficult problem of tapping.

The entropy is defined in the usual way,

$$
S=-\sum_{\boldsymbol{m}} P_{s}(\boldsymbol{m}) \ln P_{S}(\boldsymbol{m})=\frac{N}{X} D_{0}^{s}+\ln Z,
$$

which is an extensive quantity. The specific entropy per site is

$$
\begin{aligned}
\sigma & \equiv \frac{S}{N}=\frac{1}{X} D_{0}^{S}+\ln \zeta \\
& =\frac{\left(1+4 e^{-1 / X}\right)^{1 / 2}-1}{2 X\left(1+4 e^{-1 / X}\right)^{1 / 2}}+\ln \frac{1+\left(1+4 e^{-1 / X}\right)^{1 / 2}}{2} .
\end{aligned}
$$

It is possible to define a function analogous to Helmholtz's specific free energy,

$$
\Phi=-X \ln \zeta=D_{0}^{s}-X \sigma
$$

so that Eq. (5.9) can be expressed

$$
D_{0}^{s}=\frac{d(\Phi / X)}{d(1 / X)} .
$$

The description of the steady state of the TIM and that of the tapped 1SFM presented in Ref. [19] are closely related. In both models, the metastable states are the same, namely, those characterized by having all the holes isolated, i.e., surrounded by two particles. Moreover, in the weak tapping limit, the steady state probability distribution has the canonical form in both models. This implies that their "thermodynamical" properties are the same, when expressed in terms of the compactivity. Probably, this equivalence does not hold for stronger tapping, for which the simple description for the steady state developed here seems to need some refinements, as it follows from the numerical experiments reported in Refs. [24,27]. Finally, let us note that the number of holes must be even in the TIM, while the 1SFM is free from this restriction. Of course, this difference becomes irrelevant in the thermodynamic limit, in which the density of holes is a continuous variable.

\section{A. Spatial correlations}

In the steady state, the only spatial correlations present in the model are due to the impossibility of having two nearest 
neighbor holes. This property can be used to simplify the calculation of the correlation functions in the steady state.

We introduce two "entities," $\alpha$ and $\beta$, being $\alpha$ the set formed by a hole with the particle that is at its right nearest neighbor site, and $\beta$ a single particle. Thus, any metastable configuration is obtained as an, unrestricted, arbitrary permutation of the entities $\alpha$ and $\beta$. Here we take a very large system, so that we do not need to consider periodic boundary conditions. We will denote by $N_{\alpha}$ and $N_{\beta}$ the number of entities $\alpha$ and $\beta$ in a given configuration, respectively. Therefore,

$$
2 N_{\alpha}+N_{\beta}=N
$$

It is obvious that $N_{\alpha}$ equals the number of holes $k$ in the configuration, so that the density of entities $\alpha$ and $\beta$ are related to the density of holes $D_{0}$,

$$
\begin{gathered}
x_{\alpha} \equiv \frac{\left\langle N_{\alpha}\right\rangle}{N}=D_{0}, \\
x_{\beta} \equiv \frac{\left\langle N_{\beta}\right\rangle}{N}=1-2 x_{\alpha}=1-2 D_{0},
\end{gathered}
$$

where the averages are done over the considered ensemble of systems.

Since the positions of entities $\alpha$ and $\beta$ are independent in the steady state, it is very easy to compute stationary correlations. For instance, the probability of finding two holes separated by $r$ particles is given by

$$
F_{r}^{s}=\left\langle m_{k}\left(1-m_{k+1}\right) \cdots\left(1-m_{k+r}\right) m_{k+r+1}\right\rangle_{s} \equiv x_{\alpha \beta^{r-1} \alpha}^{s} .
$$

Here, $x_{\alpha \beta^{r-1} \alpha}^{s}$ is the steady density of clusters composed by two entities $\alpha$ separated by $r-1$ entities $\beta$. The number of entities $\beta$ is $r-1$ because the particle in site $k+1$ together with the hole in site $k$ constitute the first entity $\alpha$. We will consider that $r \geqslant 1$, since the holes are isolated in the metastable states. It is

$$
x_{\alpha \beta^{r-1} \alpha}=\frac{\left\langle N_{\alpha \beta^{r-1} \alpha}\right\rangle}{N},
$$

where $N_{\alpha \beta^{r-1} \alpha}$ is the number of clusters of the kind indicated above. By definition,

$$
\left\langle N_{\alpha \beta^{r-1} \alpha}\right\rangle=\left\langle N_{\alpha}\right\rangle P\left(\beta^{r-1} \alpha \mid \alpha\right),
$$

where $P\left(\beta^{r-1} \alpha \mid \alpha\right)$ is the conditional probability of finding a cluster composed of $r-1$ consecutive entities $\beta$ and one entity $\alpha$ to the right of one entity $\alpha$. As the entities $\alpha$ and $\beta$ are independent in the steady state, the stationary value of this conditional probability is

$$
P_{s}\left(\beta^{r-1} \alpha \mid \alpha\right)=P_{s}\left(\beta^{r-1} \alpha\right)=\left[P_{\beta}^{s}\right]^{r-1} P_{\alpha}^{s},
$$

where $P_{\alpha}^{s}$ and $P_{\beta}^{s}$ are the probabilities of finding an $\alpha$ and a $\beta$ entity in the steady state, respectively. Obviously,

$$
\begin{gathered}
P_{\alpha}^{s}=\frac{\left\langle N_{\alpha}\right\rangle^{s}}{\left\langle N_{\alpha}+N_{\beta}\right\rangle^{s}}=\frac{D_{0}^{s}}{1-D_{0}^{s}}, \\
P_{\beta}^{s}=\frac{\left\langle N_{\beta}\right\rangle^{s}}{\left\langle N_{\alpha}+N_{\beta}\right\rangle^{s}}=\frac{1-2 D_{0}^{s}}{1-D_{0}^{s}},
\end{gathered}
$$

where we have made use of Eq. (5.17). Therefore, putting together Eqs. (5.18)-(5.22), we get

$$
F_{r}^{s}=\frac{\left(D_{0}^{s}\right)^{2}}{1-2 D_{0}^{s}}\left(\frac{1-2 D_{0}^{s}}{1-D_{0}^{s}}\right)^{r} \quad(r \geqslant 1) .
$$

Since the moment $F_{r}^{s}$ equals the probability of finding two holes separated by $r$ particles, it is clear that

$$
F_{0}^{s}=0 \text {, }
$$

reflecting that two holes must always be separated by at least one particle. The moments $F_{r}$ obey the following "sum rule,"

$$
\sum_{r=0}^{\infty} F_{r}=D_{0}
$$

expressing that the sum of the probabilities of finding two holes separated by an arbitrary number of particles equals the probability of finding one hole, i.e.,

$$
\begin{aligned}
D_{0} & =\left\langle m_{k}\right\rangle=\underbrace{\left\langle m_{k} m_{k+1}\right\rangle}_{F_{0}}+\left\langle m_{k}\left(1-m_{k+1}\right)\right\rangle \\
& =F_{0}+F_{1}+\left\langle m_{k}\left(1-m_{k+1}\right)\left(1-m_{k+2}\right)\right\rangle \\
& =\cdots=\sum_{r=0}^{\infty} F_{r} .
\end{aligned}
$$

The calculation of other spatial correlations in the steady state is straightforward, by following a line of reasoning similar to the one used to find $F_{r}^{s}$. For instance, $F_{r}^{s}$ also provides the probability of finding a cluster composed by two entities $\alpha$ and $r-1$ entities $\beta$, no matter the way they are ordered, because of the independence of the entities $\alpha$ and $\beta$ in the steady state.

\section{FINAL REMARKS}

In this paper we have analyzed a one-dimensional Ising model with nearest neighbor interactions formulated in a way appropriated for the study of compaction in granular media. An equivalent particle-hole description has been introduced, in which the holes are associated to the domain walls of the original Ising system. The free relaxation of the system is modeled by a $T=0$ dynamics [13], which only allows those spin flips decreasing the energy of the system. Any configuration with all the holes (domain walls) being isolated is metastable, i.e., it does not evolve with this $T$ $=0$ dynamics. The tapping process is described as composed of two steps: (a) vibration, i.e., starting from a metastable 
configuration, each spin of the system is flipped with probability $p$, and (b) the system freely relaxes with the $T=0$ dynamics until it reaches a, in general, different metastable configuration. The parameter characterizing the tapping process is the "vibration intensity" $p$.

In the particle-hole description, the dynamics at $T=0$ is analytically solvable, by writing a closed hierarchy of equations for the probability distribution functions $D_{r}$ of finding $r+1$ consecutive holes in the system [18]. In the long time limit, the system gets stuck in a state where $D_{r}=0$ for all $r>1$, i.e., all the holes are isolated, as indicated above.

Tapping is a rather complex process, since each tap is composed of two neatly different processes: vibration and free relaxation. In order to get a physical insight into the mechanisms responsible for the behavior of the system under tapping, the derivation of the effective transition probabilities for the Markov process connecting the metastable states reached by the system in two consecutive taps is needed. In general, this is a formidable task, but in the limit of a gently tapped system these transition rates between metastable states can be computed up to the second-order in $p$.

In the first order, the only possible transitions are diffusion and destruction of a hole pair. Then, compaction takes place, since there are no processes decreasing the density of particles in the system to the lowest order. To describe the steady state, second-order processes must be taken into account, so as to have transitions that increase the number of holes. Interestingly, these transitions are just the inverse of those decreasing the number of holes to the lowest order. Therefore, the Markov process is irreducible, i.e., all the metastable configurations are connected through a chain of transitions with nonzero probability. As a consequence, there is an unique, well-defined, steady probability distribution for each value of $p$. Besides, the effective transition rates verify detailed balance. This property has been used to derive the steady distribution analytically, finding that it has the canonical form. Thus, a relationship between Edwards' compactivity and the vibration intensity $p$ is obtained, in the limit of weak tapping.

The system analyzed in this paper, as formulated for modeling tapped granular media, is closely related to the onedimensional facilitated Ising model $[10,19,23]$. In the respective particle-hole pictures, the metastable states are the same, those having all the holes isolated. Although, in the limit of weak tapping, the corresponding effective dynamics connect- ing metastable configurations are not equivalent, the steady state is described in both cases by the canonical distribution. The role of the energy is played by the number of holes and that of the temperature by Edwards' compactivity, which is related to the vibration intensity by an Arrhenius-like expression. In this way, we find a sort of "minimal" model for Edwards' description of the steady state of externally perturbed granular media: a one-dimensional system of particles and holes, with the metastable states characterized by having all the holes isolated, and a canonical probability distribution function. Nevertheless, in order to have an actually complete description of the steady state, a relationship between the parameters characterizing the statics and the dynamics of the system, i.e., between the compactivity and the vibration intensity, is needed. Then, it is also necessary to derive the effective dynamics from the underlying original models (TIM, 1SFM, etc.), when trying to understand the steady state behavior.

The effective dynamics approach between metastable states has been shown to be a powerful tool, in order to study the steady state of models for granular systems submitted to tapping processes. It allows to identify the physical mechanisms responsible for the increase of the density, and also for the existence of a steady state characterized by a density being a monotonic decreasing function of the vibration intensity. In simple models, the calculations can be thoroughly done in the limit of gentle tapping, deriving analytically the steady state distribution. The results so obtained are consistent with recent extensive numerical tests of Edwards' hypothesis in simple systems [13,24-27], although the systematic deviations found for stronger tapping [24,27] cannot be accounted for within the second-order theory developed in this paper and in Ref. [19]. This would need an extension of the effective dynamics approach to the whole range of vibration intensities, which is certainly not an easy task. Nevertheless, it is hard to believe that the simple structure of the transition probabilities of the effective dynamics should remain unaltered for stronger tapping, i.e., deviations from the simple canonical distribution found here are to be expected.

\section{ACKNOWLEDGMENT}

We acknowledge support from the Ministerio de Ciencia y Tecnología (Spain) through Grant No. BFM2002-00303 (partially financed by FEDER funds).
[1] H.M. Jaeger, S.R. Nagel, and R.P. Behringer, Rev. Mod. Phys. 68, 1259 (1996).

[2] L.P. Kadanoff, Rev. Mod. Phys. 71, 435 (1999).

[3] J.B. Knight, C.G. Frandich, C.N. Lau, H.M. Jaeger, and S.R. Nagel, Phys. Rev. E 51, 3957 (1995).

[4] E.R. Nowak, J.B. Knight, M. Povinelli, H.M. Jaeger, and S.R. Nagel, Powder Technology 94, 79 (1997).

[5] E.R. Nowak, J.B. Knight, E. Ben-Naim, H.M. Jaeger, and S.R. Nagel, Phys. Rev. E 57, 1971 (1998).

[6] H. M. Jaeger, in Physics of Dry Granular Media, edited by H.
J. Herrmann et al. (Kluwer Academic, Dordrecht, 1998), p. 553.

[7] E. Caglioti, V. Loreto, H.J. Herrmann, and M. Nicodemi, Phys. Rev. Lett. 79, 1575 (1997).

[8] M. Nicodemi, A. Coniglio, and H.J. Herrmann, Phys. Rev. E 55, 3962 (1997).

[9] E. Ben-Naim, J.B. Knight, E.R. Nowak, H.M. Jaeger, and S.R. Nagel, Physica D 123, 380 (1998).

[10] J.J. Brey, A. Prados, and B. Sánchez-Rey, Phys. Rev. E 60, 5685 (1999). 
[11] J. Berg and A. Mehta, Adv. Complex Syst. 4, 309 (2001).

[12] P.F. Stadler, J.M. Luck, and A. Mehta, Europhys. Lett. 57, 46 (2002).

[13] A. Lefevre and D.S. Dean, J. Phys. A 34, L213 (2001).

[14] D.S. Dean and A. Lefevre, Phys. Rev. Lett. 86, 5639 (2001).

[15] J. Berg and M. Sellitto, Phys. Rev. E 65, 016115 (2001).

[16] J. Berg and A. Mehta, Phys. Rev. E 65, 031305 (2002).

[17] N. G. van Kampen, Stochastic Processes in Physics and Chemistry (North-Holland, Amsterdam, 1992).

[18] A. Prados and J.J. Brey, J. Phys. A 34, L453 (2001).

[19] J.J. Brey, A. Prados, and B. Sánchez-Rey, Physica A 275, 310 (2000).

[20] G.H. Fredrickson and H.C. Andersen, Phys. Rev. Lett. 53, 1244 (1984).

[21] S.F. Edwards and R.B.S. Oakeshott, Physica A 157, 1080 (1989).
[22] A. Mehta and S.F. Edwards, Physica A 157, 1091 (1989).

[23] A. Prados, J.J. Brey, and B. Sánchez-Rey, Physica A 284, 277 (2000).

[24] J. Berg, S. Franz, and M. Sellitto, Eur. Phys. J. B 26, 349 (2002).

[25] A. Barrat, J. Kurchan, V. Loreto, and M. Sellitto, Phys. Rev. Lett. 85, 5034 (2000).

[26] A. Barrat, J. Kurchan, V. Loreto, and M. Sellitto, Phys. Rev. E 63, 051301 (2001).

[27] A. Lefevre, e-print cond-mat/0202376.

[28] H.A. Makse and J. Kurchan, Nature (London) 415, 614 (2002).

[29] A. Crisanti, F. Ritort, A. Rocco, and M. Sellito, J. Chem. Phys. 113, 10615 (2000).

[30] R.J. Glauber, J. Math. Phys. 4, 294 (1963).

[31] E. Follana and F. Ritort, Phys. Rev. B 54, 930 (1996). 